\title{
Ultrahigh Energy Cosmic Rays: Old Physics or New Physics?
}

\author{
F.W. Stecker \\ Laboratory for High Energy Astrophysics \\ NASA Goddard Space Flight Center, Greenbelt, MD, USA
}

\begin{abstract}
We consider the advantages of and the problems associated with hypotheses to explain the origin of ultrahigh energy cosmic rays (UHECR: $E>10 \mathrm{EeV}$ ) and the "trans-GZK" cosmic rays (TGZK: $E>100 \mathrm{EeV}$ ) both through "old physics" (acceleration in cosmic sources) and "new physics" (new particles, topological defects, fat neutrino cross sections, Lorentz invariance violation).
\end{abstract}

\section{Introduction}

Owing to their observed isotropy (e.g., Stokes, these proceedings), and ultrahigh energy, cosmic rays above $10 \mathrm{EeV}\left(1 \mathrm{EeV} \equiv 10^{18} \mathrm{eV}\right)$ are believed to be of extragalactic origin. Shortly after the discovery of the $3 \mathrm{~K}$ cosmogenic background radiation (CBR), Greisen (1966) and Zatsepin and Kuz'min (1966) predicted that pion-producing interactions of such cosmic ray protons with the CBR should produce a spectral cutoff at $E \sim 50 \mathrm{EeV}$ (the GZK cutoff). The GZK effect is not a true cutoff, but a suppression of the ultrahigh energy cosmic ray flux owing to an energy dependent propagation time against energy losses by such interactions, a time which is only 300 Myr for 100 EeV protons (Stecker 1968). At high redshifts, $z$, the target photon density increases by $(1+z)^{3}$ and both the photon and initial cosmic ray energies increase by $(1+z)$. A plot of the GZK energy as a function of redshift, calculated for the $\Lambda$ CDM cosmology, is shown in Figure 1 (Stecker and Scully 2004). If the source spectrum is hard enough, there could also be a relative enhancement just below the "GZK energy" owing to a "pileup" of cosmic rays starting out at higher energies and crowding up in energy space at or below the predicted cutoff energy. At energies in the 1-10 EeV range, pair production interactions should take a bite out of the UHECR spectrum. 


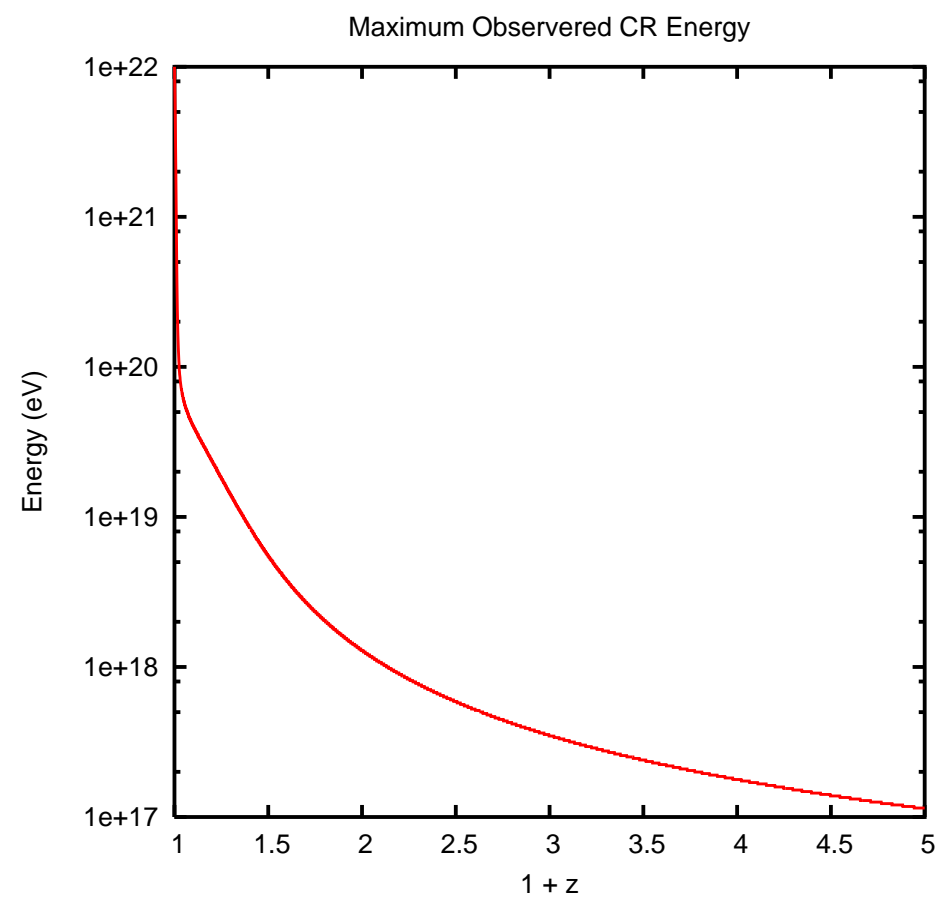

Fig. 1. The GZK cutoff energy, defined as the energy predicted for a flux decrease of $1 / e$ owing to intergalactic photomeson production interactions, as a function of redshift (Stecker and Scully 2004).

UHECRs produce giant air showers. Observational studies of these showers have been done with scintillator arrays and with atmospheric flourescence detectors. The AGASA scintillator array collaboration claims a significant number of events at trans-GZK energies (Teshima, these proceedings). However, HiRes monocular data obtained using the flourescence technique appear to be consistent with the GZK effect (Zech, these proceedings). The AGASA data indicate a deviation from the predicted GZK effect, even if the number density of ultrahigh energy sources is weighted like the local galaxy distribution (Blanton, et al. 2001). De Marco et al. (2003) have argued that the discrepency between the AGASA and HiRes results is not statistically significant. Better statistics will require data from future ground based detectors such as Auger (Suomijarvi, Privatera, and Perrone, these proceedings) and space based detectors such as $O W L$ (Stecker, elsewhere in these proceedings) and EUSO (D'Ali'Stati, these proceedings).

The Auger project now under construction will use both scintillators and 
fluorscence detectors so that combined results from Auger can help clarify the present prima facie discrepency between the AGASA and HiRes results obtained using these different techiques.. (Note that a fluorescence detector such as HiRes, namely Fly's Eye, reported the highest energy event yet seen, it viz., $E \simeq 300 \mathrm{EeV}$.) It is apparent that the observational situation is interesting enough and the physics implications are important enough to justify both more sensitive future detectors and the theoretical investigation of new physics and astrophysics. The significance of a non-observation of a GZK effect is profound. Such a result either requires a large overdensity of UHECRs within about $100 \mathrm{Mpc}$ emitted by unidentified "local" sources and trapped by magnetic fields, or it requires new physics.

\section{Old Physics: The "Bottom-Up" Scenario}

The apparent lack of a GZK cutoff (with the exception of the new HiRes results) has led astrophysicists to hunt for nearby cosmic "zevatrons" which can accelerate particles to energies $\mathcal{O}\left(1 \mathrm{ZeV} \equiv 10^{21} \mathrm{eV}\right)$. It is generally assumed that the diffusive shock acceleration process is the most likely mechanism for accelerating particles to high energy in astrophysical sources. In this case, the maximum obtainable energy is given by $E_{\max }=k e Z(u / c) B L$, where $u \leq$ $c$ is the shock speed, $e Z$ is the charge of the particle being accelerated, $B$ is the magnetic field strength, $L$ is the size of the accelerating region and the numerical parameter $k=\mathcal{O}(1)$. Taking $k=1$ and $u=c$, one finds $E_{\text {max }}=0.9 Z(B L)$, with $E$ in $\mathrm{EeV}, B$ in $\mu \mathrm{G}$ and $L$ in kpc. This assumes that particles can be accelerated efficiently up until the moment when they can no longer be contained by the source, i.e., until their gyroradius becomes larger than the size of the source. There are not many cosmic zevatron candidates. Galactic sources such as white dwarfs, neutron stars, pulsars, and magnetars can be ruled out because their galactic distribution would lead to UHECR anisotropies and this is not the case. Perhaps the most promising potential zevatrons are radio lobes of strong radio galaxies (Biermann and Strittmatter 1987). The trick is that such sources need to be found close enough to avoid

the GZK cutoff. For example, the nearby radio galaxy M87 may be a source of observed trans-GZK cosmic rays (Stecker 1968; Farrar and Piran 2000). Such an explanation would require one to invoke magnetic field configurations capable of producing a quasi-isotropic distribution of trans-GZK protons with energies > $100 \mathrm{EeV}$, making this hypothesis questionable. However, if the primary particles are nuclei (see Section 2.1), it is easier to explain a radio galaxy origin for the two highest energy events (Stecker and Salamon 1999). 


\subsubsection{The Dead Quasar Origin Hypothesis}

All large galaxies are suspected to harbor supermassive black holes in their centers which may have once been quasars, fed by accretion disks which are now used up. It has been suggested that nearby quasar remnants may be the searched-for zevatrons (Boldt and Ghosh 1999; Boldt and Lowenstein 2000). This scenario also has potential theoretical problems and needs to be explored further. In particular, it has been shown that black holes which are not accreting plasma cannot possess a large scale magnetic field with which to accelerate particles to relativistic energies (Ginzburg and Ozernoi 1964; Krolik 1999). Observational evidence also indicates that the cores of weakly active galaxies have low magnetic fields (Falcke 2001 and references therein).

\subsubsection{The Cosmological Gamma-Ray Burst Origin Hypothesis}

It has also been suggested that cosmological $\gamma$-ray bursts (GRBs) could be the zevatron sources of the highest energy cosmic rays if these objects emitted the same amount of energy in ultrahigh energy $\left(\sim 10^{14} \mathrm{MeV}\right)$ cosmic rays as in $\sim$ $\mathrm{MeV}$ photons (Waxman 1995; Vietri 1995). However, 26 of the 27 bursts with identified host galaxies as of 2003 are at moderate to high redshifts $(z>0.36)$, with GRB00013 having a redshift of 4.50; they are not nearby sources.

The host galaxies of GRBs are sites of very active star formation (Christensen, et al. 2004). The bursts occur within star forming regions. The GRB redshift distribution follows the strong redshift evolution of the cosmic star formation rate (Schmidt 2001: Stern et al. 2002) with a much lower burst rate at the low redshifts from which the TGZK events must come. GRBs are thought to be supernovae caused by the core collapse of massive stars (Cherepashchuk and Postnov 2001) and the core collapse supernova rate rate at $z=0.26$ has been found to be a factor of $\sim 3$ higher than the estimate for $z=0$ (Cappellaro, et al. 2004). There is also some evidence for luminosity evolution; GRBs may have been brighter at higher redshifts (Amati 2004). Schmidt (2001) concludes that the local $(z=0)$ total energy release rate by all GRBs in the $\gamma$-ray range is $\mathcal{O}\left(10^{28}\right) \mathrm{W} \mathrm{Mpc}^{-3}$. whereas the required energy input rate in UHECRs above $10 \mathrm{EeV}$ is $\mathcal{O}\left(10^{31}\right) \mathrm{W} \mathrm{Mpc}^{-3}$. GRBs fail by at least an order of magnitude to account for TGZK ( $>100 \mathrm{EeV}$ ) events (Stecker 2000) and they fail by at least two orders of magnitude to account for the UHECR $(>10 \mathrm{EeV}$ ) events (Scully and Stecker 2002).

Norris (2002) has given an analysis of the luminosities and space densities of nearby low luminosity long-lag GRB sources which are identified with Type I supernovae. For these sources, he finds a rate per unit volume of $7.8 \times 10^{-7}$ $\mathrm{Mpc}^{-3} \mathrm{yr}^{-1}$ and an average (isotropic) energy release per burst of $1.3 \times 10^{49}$ erg over the energy range from 10 to $1000 \mathrm{keV}$. The energy release per unit 
volume is then $\sim 3 \times 10^{28} \mathrm{~W} \mathrm{Mpc}^{-3}$, more than two orders of magnitude below the rate needed to account for the TGZK events. Even these numbers are most likely too optimistic, since they are based on the questionable assumption of the same amount of GRB energy being put into ultrahigh energy cosmic rays as in $\sim \mathrm{MeV}$ photons.

\subsection{The Heavy Nuclei Origin Scenario}

A more conservative hypothesis for explaining the trans-GZK events is that they were produced by heavy nuclei. The conditions under which they were accelerated in astrophysical sources would have to preclude dissociation. Stecker and Salamon (1999) have shown that the energy loss time for nuclei starting out as $\mathrm{Fe}$ is longer than that for protons for energies up to a total energy of $\sim 300 \mathrm{EeV}$ (see Fig. 2). Stanev et al. (1995) and Biermann (1998) have examined the arrival directions of the highest energy events. They point out that the $\sim 200 \mathrm{EeV}$ event is within $10^{\circ}$ of the direction of the strong radio galaxy NGC 315. This galaxy lies at a distance of only $\sim 60 \mathrm{Mpc}$ from us. For that distance, the results of Stecker and Salamon (1999) indicate that heavy nuclei would have a cutoff energy of $\sim 130 \mathrm{EeV}$, which may be within the uncertainty in the energy determination for this event. The $\sim 300 \mathrm{EeV}$ event is within $12^{\circ}$ of the direction of the strong radio galaxy $3 \mathrm{C} 134$. The distance to $3 \mathrm{C} 134$ is unfortunately unknown because its location behind a dense molecular cloud in our own galaxy obscures the spectral lines required for a measurement of its redshift. A clue that we may be seeing heavier nuclei above the proton-GZK cutoff comes from a recent analysis of inclined air showers above $10 \mathrm{EeV}$ energy (Ave, et al. 2000). These results favor proton primaries below the p-GZK cutoff energy but they appear to favor a heavier composition above the $p$-GZK cutoff energy. We note that continuation of the UHECR spectrum to energies significantly above $300 \mathrm{EeV}$ would rule out heavy nuclei (Stecker and Salamon 1999).

\section{New Physics}

The existence of TGZK events, as well as the difficulty in finding reasonable candidates for zevatrons, has stimulated theorists to look for possible solutions involving new physics. Some of these involve (A) Top-down scenarios involving such concepts as grand unification and early universe physics, (B) a large increase in the neutrino-nucleon cross section at ultrahigh energies, (C) new particles, and (D) a small violation of Lorentz invariance. It should also be noted that, even if the GZK effect is seen, top-down scenarios predict the reemergence of a new component at even higher energies (Bhattacharjee and 


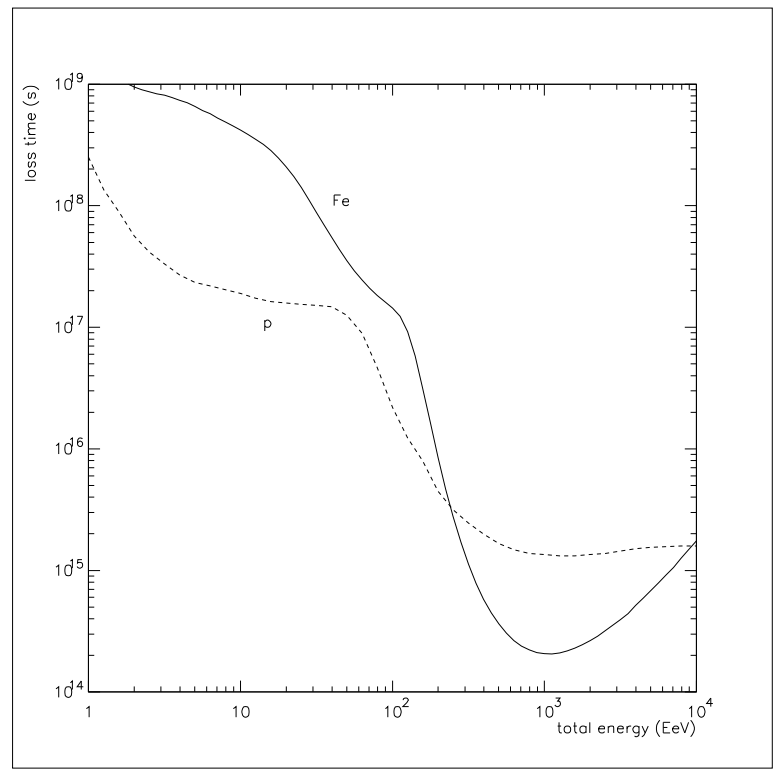

Fig. 2. Mean energy loss times for protons and nuclei originating as iron (Stecker and Salamon 1999).

Sigl 2000).

\subsection{Top-Down Scenarios: "Fraggers"}

A way to avoid the problems with finding plausible astrophysical zevatrons is to start at the top, i.e., the energy scale associated with grand unification theories (GUTs), supersymmetric grand unification (SUSY-GUTs) and superstring theory unification. In the very early stages of the big bang, the universe is believed to have reached temperatures appropriate to unification theories. Very heavy "topological defects" may be produced as a consequence of the GUT phase transition when the strong and electroweak forces became separated. Topological defects in the vacuum of space are caused by misalignments of the heavy Higgs fields in regions which were causally disconnected in the early history of the universe. These are localized regions where extremely high densities of mass-energy consisting of early universe Higgs fields are trapped. Such defects go by designations such as cosmic strings, monopoles, walls, necklaces (strings bounded by monopoles), and textures, depending on their geometrical and topological properties. Superheavy particles or topological structures arising at the GUT energy scale $M \geq 10^{5} \mathrm{EeV}$ can decay or annihilate to produce " $X$-particles" (GUT scale Higgs particles, superheavy fermions, or leptoquark bosons of mass M.) These $X$-particles will decay to produce QCD fragmentation jets at ultrahigh energies. I will call such sources "fraggers". Fraggers produce mainly pions, with a 3 to 10 per cent admixture of baryons, 
so that generally one can expect them to produce more high energy $\gamma$-rays and $\nu$ 's than protons. The number of GUT and SUSY-GUT top-down models is quite large (Bhattacharjee and Sigl 2000).

\section{1 .1 "Z-bursts"}

In principle, ultra-ultrahigh energy $\mathcal{O}(10 \mathrm{ZeV})$ neutrinos can produce ultrahigh energy $Z$-boson fraggers by interactions with $1.9 \mathrm{~K}$ thermal CBR neutrinos (Weiler 1982) resulting in " $Z$-burst" fragmentation jets. This will occur at the resonance energy $E_{\text {res }}=4\left[m_{\nu}(\mathrm{eV})\right]^{-1} \mathrm{ZeV}$. A typical $Z$ boson will decay to produce $\sim 2$ nucleons, $\sim 20 \gamma$-rays and $\sim 50$ neutrinos, $2 / 3$ of which are $\nu_{\mu}$ 's. If the nucleons produced from $Z$-bursts originate within a few tens of Mpc of the Earth they can reach us, even though the original $\sim 10 \mathrm{ZeV}$ neutrinos could have come from a much further distance. It has been suggested that this effect can be amplified if our galaxy has a halo of neutrinos with a mass of tens of eV (Fargion, et al. 1999; Weiler 1999). However, a neutrino mass large enough to be confined to a galaxy size neutrino halo (Tremaine and Gunn 1979) or even a galaxy cluster size halo (Shafi and Stecker 1984) is now clearly ruled out by the results of the Wilkonson Microwave Anisotropy Probe (WMAP). These results, combined with other cosmic microwave background data and data from the $2 \mathrm{dF}$ galaxy redshift survey, together with the very small neutrino flavor mass differences implied by the atmospheric and solar neutrino oscillation results, indicate that even the heaviest neutrino would have a mass in the sub-eV range, i.e., $0.03 \mathrm{eV} \leq m_{3} \leq 0.24 \mathrm{eV}$ (Bhattacharyya et al. 2003; Allen et al. 2003). The tritium decay spectral endpoint limits on the mass of the $\nu_{e}$ are also consistent with this conclusion. Thus, neutrino masses are too small for halo or galaxy cluster confinement.

The severe problem with the $Z$-burst explanation for the TGZK events is that one needs to produce large fluxes of neutrinos with energies in excess of $10 \mathrm{ZeV}$. If these are secondaries from pion production, the primary protons which produce them must have energies of hundreds of $\mathrm{ZeV}$ ! We know of no source capable of accelerating particles such energies. A more likely process to produce $10 \mathrm{ZeV}$ neutrinos would be via top-down fraggers. The flux of such neutrinos is constrained because the related energy release into electromagnetic cascades which produce $\mathrm{GeV}$ range $\gamma$-rays is limited by satellite observations (Bhattacharjee and Sigl 2000). This constraint, together with the

low probability for $Z$-burst production rule out this scenario for explaining the TGZKs. 


\subsubsection{Superheavy Dark Matter Particles}

The inflation of the early universe in the accepted big-bang model is postulated to be caused by a putative vacuum field called the inflaton field. During inflation, the universe is cold but, when inflation is over, coherent oscillations of the inflaton field reheat it to a high temperature. While the inflaton field is oscillating, non-thermal production of very heavy particles may take place. These heavy particles may survive to the present as dark matter. They are also fraggers. Their decays or annihilation will produce ultrahigh energy particles and photons via fragmentation. It has been suggested that such particles may be the source of ultrahigh energy cosmic rays (Berezinsky et al. 1997; Kuz'min and Rubakov 1998; Blasi et al. 2002; Sarkar and Toldrà 2002; Barbot and Drees 2002). A comparison of recent experimental constraints from dark matter nuclear recoil searches with predicted rates gives a lower limit on the mass of superheavy dark matter particles of $10^{6} \mathrm{EeV}$, unless they interact weakly with normal matter (Albuquerque amd Baudis 2003). The annihilation or decay of such particles in a dark matter halo of our galaxy would produce ultrahigh energy nucleons which would not be attenuated at TGZK energies owing to their proximity. The resulting air shower distribution would then be anisotropic. This would be an even larger effect in the case of annihilation than decay, since the flux would then scale as the square of the particle density density rather than linearly. Since the galactic center is viewed from the southern hemisphere, the location of the AUGER detector will make it ideal for testing this hypothesis.

\subsubsection{Halo Fraggers and the Missing Photon Problem}

Halo fragger models such as $Z$-burst and superheavy halo dark matter decay or annihilation will produce more ultrahigh energy photons than protons. These ultrahigh energy photons can reach the Earth from anywhere in a dark matter galactic halo because there is a "mini-window" for the transmission of ultrahigh energy cosmic rays between $\sim 0.1$ and $\sim 10^{6} \mathrm{EeV}$ (Stecker 2003). Such photon-induced giant air showers have an evolution profile which is significantly different from nucleon-induced showers because of the LandauPomeranchuk-Migdal effect and also cascading in the Earth's magnetic field (Cillis et al. 1999). Taking this into account, Shinozaki, et al. (2002) have used the AGASA data to place upper limits on the primary photon composition of their UHECR events. They find an initial photon fraction upper limit of $28 \%$ for events above $10 \mathrm{EeV}$ and $67 \%$ for events above $30 \mathrm{EeV}$ at a $95 \%$ confidence level with no indication of photonic showers above $100 \mathrm{EeV}$. A recent reanalysis of the ultrahigh energy events observed at Haverah Park by Ave, et al. (2000) indicates that less than half of the events (at 95\% confidence level) observed above 10 and $40 \mathrm{EeV}$ are $\gamma$-ray initiated. An analysis of the highest energy Fly's Eye event $(E=300 \mathrm{EeV})$ shows it not to be of photonic origin 
(Halzen and Hooper 2002).

In order to solve the missing photon problem for halo fraggers, Chisholm and Kolb (2004) have suggested that a small violation of Lorentz invariance could allow ultrahigh energy photons to decay into electron-positron pairs (Coleman and Glashow 1999), thus eliminating the photon component of the fragger-produced flux. The amount of Lorentz invariance required is within the observational limits obtained by Stecker and Glashow (2001). However, the scenario suggested by Chisholm and Kolb, implies that neutrons would be the primary ultrahigh particles producing the giant air showers, again producing a halo anisotropy for which there is no present indication (Shinozaki, et al. 2002; Kachelrieß and Semikoz 2003).

\subsection{Increasing the Neutrino-Nucleon Cross Section at Ultrahigh Energies}

Various processes have been invoked to produce observable fluxes of high energy neutrinos (see, e.g., Stecker 2003). Since neutrinos can travel through the universe without interacting with the $2.7 \mathrm{~K} \mathrm{CBR}$, it has been suggested that if the neutrino-nucleon cross section were to increase to hadronic values at ultrahigh energies, they could produce the giant air showers and account for the observations of showers above the proton-GZK cutoff (see, e.g., Ringwald, these proceedings).

Several suggestions have been made for processes that can enhance the neutrinonucleon cross section at ultrahigh energies. These suggestions include composite models of neutrinos (Domokos and Nussinov 1987), scalar leptoquark resonance channels (Robinett 1988) and the exchange of dual gluons (Bordes, et al. 1998). Burdman, et al. (1998) have ruled out a fairly general class of these types of models by considering accelerator data and unitarity bounds. More recently, the prospect of enhanced neutrino cross sections has been explored in the context of extra dimension models invoked by some theorists as a possible way for accounting for the extraordinary weakness of the gravitational force (Arkani-Hamed, et al. 1999; Randall and Sundrum 1999). These models allow the virtual exchange of gravitons propagating in the bulk (i.e. in the space of full extra dimensions) while restricting the propagation of other particles to the familiar four dimensional space-time manifold. It has been suggested that in such models, $\sigma(\nu \mathrm{N}) \simeq\left[E_{\nu} /(100 \mathrm{EeV})\right] \mathrm{mb}$ (Nussinov and Schrock 1999; Domokos and Kovesi-Domokos 1999; Jain, et al. 2000). Other scenarios involve the neutrino-initiated atmopheric production of black holes (Anchordoqui et al. 2002; Feng and Shapere 2002) and higher dimensional extended objects, p-dimensional branes called "p-branes" (Ahn, et al. 2002; Anchordoqui, et al. 2002). Such interactions, in principle, can increase the neutrino total atmospheric interaction cross section by orders of magnitude 
above the standard model value. However, sub-mm gravity experiments and astrophysical constraints rule out total $\nu \mathrm{N}$ cross sections as large as $100 \mathrm{mb}$ as would be needed to fit the trans-GZK energy air shower profile data. Nonetheless, extra dimension models may produce significant increases in this cross section, resulting in moderately penetrating air showers. Such showers should also be present at lower energies (Anchordoqui et al. 2001; Tyler, et al. 2001). No such showers have been observed, putting an indirect constraint on extra dimension $\mathrm{TeV}$-scale gravity models.

\subsection{New Particles}

The suggestion has also been made that undiscovered neutral hadrons containing a light gluino could be producing the trans-GZK events (Farrar 1996; Cheung, et al. 1998; Berezinsky, et al. 2002). While the invocation of such particles is an intriguing idea, it seems unlikely that such particles of a few proton masses would be produced in copious enough quantities in astrophysical objects without being detected in terrestrial accelerators. There are also strong accelerator constraints on light gluino production (Alavi-Harati, et al. 1999). One should note that while it is true that the GZK threshold for such particles would be higher than that for protons, such is also the case for the more prosaic heavy nuclei previously discussed. In addition, such neutral particles cannot be accelerated directly, but must be produced as secondary particles, making the energetics reqirements more difficult.

\subsection{Violating Lorentz Invariance}

With the idea of spontaneous symmetry breaking in particle physics came the suggestion that Lorentz invariance (LI) might be weakly broken at high energies (Sato and Tati 1972). Although no true quantum theory of gravity exists, it was suggested that LI might be violated in such a theory with astrophysical consequences (Amelino-Camilia et al. 1998; Galante, these proceedings). A simple formulation for breaking LI by a small first order perturbation in the electromagnetic Lagrangian which leads to a renormalizable treatment has been given by Coleman and Glashow (1999). Using this formalism, these authors point out that different particles can have different maximum attainable velocities (MAVs) which can be different from $c$. If we denote the MAV of a particle of type $i$ by $c_{i}$ and the difference $c_{i}-c_{j} \equiv \delta_{i j}$ then Coleman and Glashow have shown that for interactions of protons with CBR photons of

energy $\epsilon$ and temperature $T_{C B R}=2.73 \mathrm{~K}$, pion production is kinematically forbidden and thus photomeson interactions are turned off if 


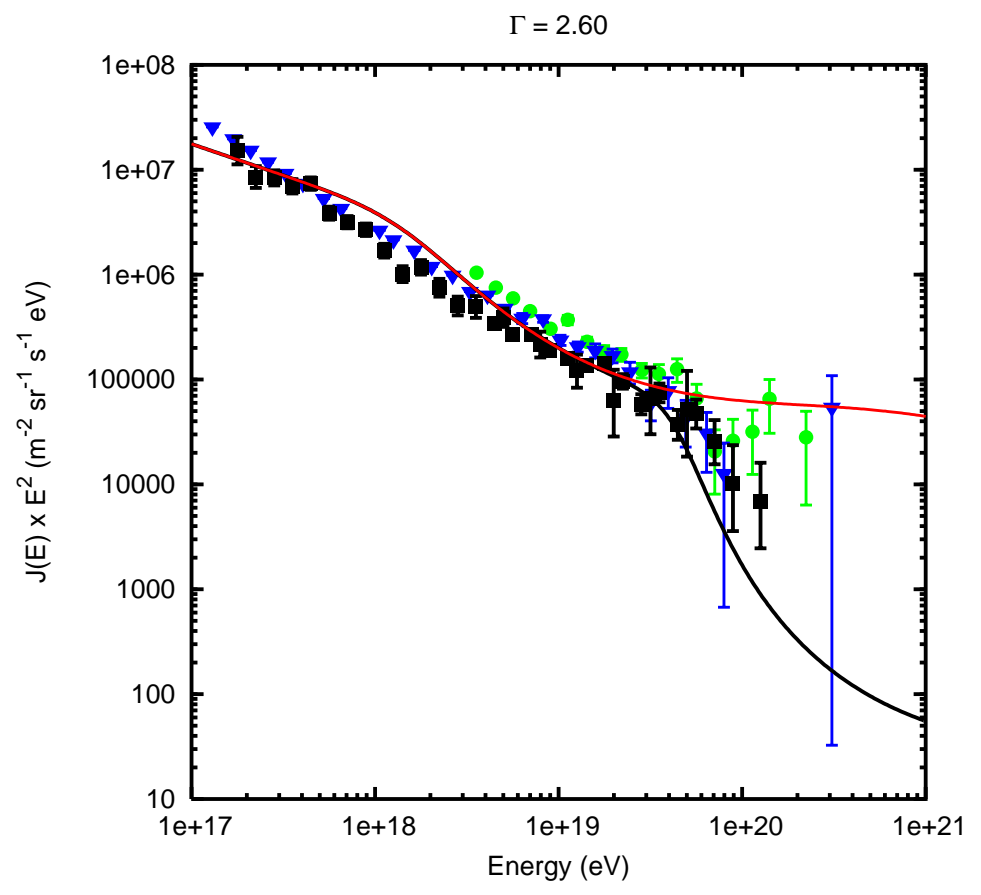

Fig. 3. Predicted spectra for an $E^{-2.6}$ source spectrum with source evolution (see text) shown with pair-production losses included and photomeson losses both included (black curve) and turned off (lighter (red) curve) (Stecker and Scully 2004). The curves are shown with ultrahigh energy cosmic ray spectral data from Fly's Eye (triangles), AGASA (circles), and HiRes monocular data (squares). They are normalized to the data at $3 \mathrm{EeV}$ (see text).

$$
\delta_{p \pi}>5 \times 10^{-24}\left(\epsilon / T_{C B R}\right)^{2} .
$$

The corrsponding condition for suppression of electron-positron pair production interactions is given by

$$
\delta_{e p}>5 \times 10^{-19}\left(\epsilon / T_{C B R}\right)^{2} .
$$

Thus, given even a very small amount of LI violation, both photomeson and pair-production interactions of UHECR with the CBR can be turned off. Such a violation of Lorentz invariance might be produced by Planck scale effects (Aloisio, et al. 2000; Alfaro and Palma 2002, 2003). The amount of LI violation 


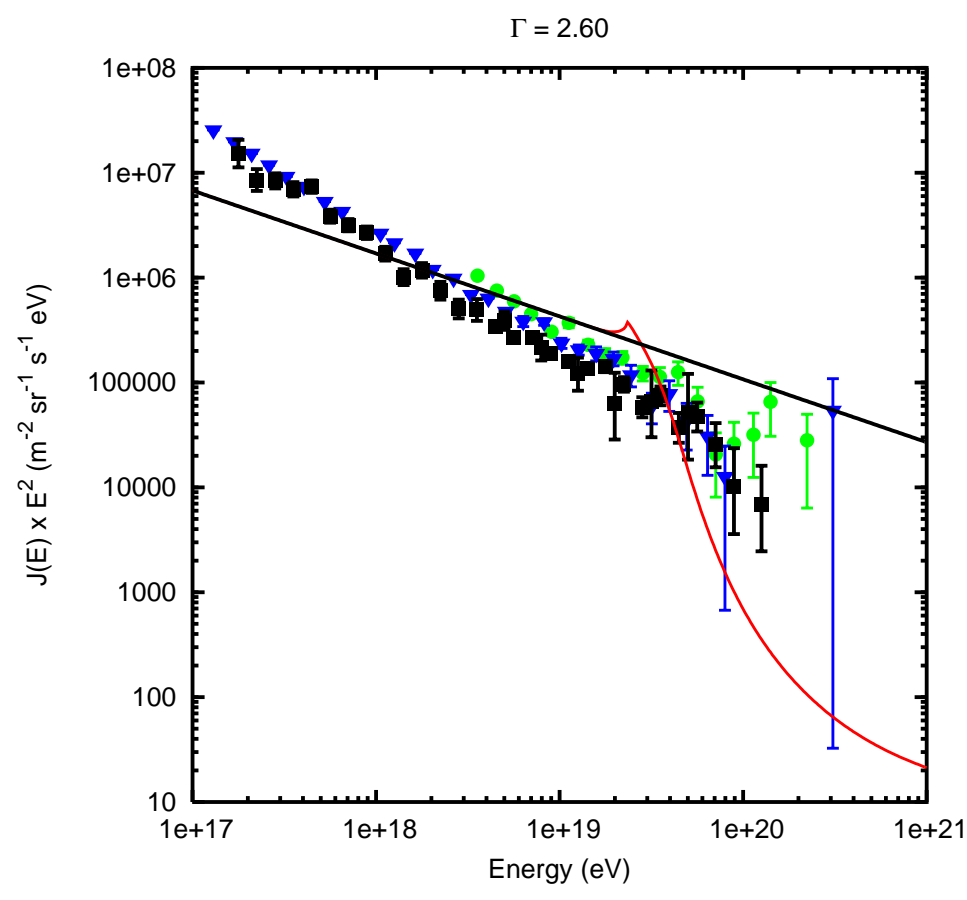

Fig. 4. Predicted spectra for an $E^{-2.6}$ source spectrum with source evolution (see text) shown with pair-production losses turned off and with photomeson production losses included (lighter (red) curve) and turned off (black straight line) (Stecker and Scully 2004). The curves are shown with ultrahigh energy cosmic ray spectral data from Fly's Eye (triangles), AGASA (circles), and HiRes monocular data (squares). They are normalized to the data at $3 \mathrm{EeV}$ (see text).

required is small compared to the constraint obtained by Stecker and Glashow (2001) from the non-suppression of intergalactic absorption of multi-TeV $\gamma$ rays, viz., $\delta_{e \gamma}<1.3 \times 10^{-15}$. The most stringent constraints to date on LI violation in QED interactions are given by Jacobson, et al. (2004).

Figures 3 and 4, show the predicted spectra obtained assuming an $E^{-2.6}$ source spectrum and a source luminosity evolution $\propto(1+z)^{3.6}$ for $0<z<2$ with no further evolution out to $z_{\max }=5$, following the star formation rate. The resulting spectra, normalized at an energy of $3 \mathrm{EeV}$ above which energy the extragalactic component is assumed to be dominant (see, e.g., Stecker 2003), are calculated for "on" and "off" energy losses for both photomeson production and pair production for protons, 
The cosmic ray spectral data from the Fly's Eye, AGASA, and HiRes detectors are also shown. ${ }^{1}$ From Figs. 3 and 4 , it can be seen that, in principle, a very small amount of LI violation can eliminate the GZK "cutoff". When pair-production is turned off, the $\sim 10 \mathrm{EeV}$ "bite" in the predicted spectrum is eliminated. Of course, when both interactions are turned off, all of the features in the predicted spectrum disappear and only a power-law remains. Contrary to the discussion of Alfaro and Palma (2003), as can be seen from the curves in Figs. 3 and 4, the present data cannot be used to put constriants on LI violation in pair-production interactions; these data appear to be consistent with either the presence or absence of such interactions. In the case of bottom-up models, there remains the problem of accelerating protons to TGZK energies in the sources (see Section 2) which, of course, is not a problem for top-down models (see Section 3 and Aloisio, these proceedings).

\section{Distinguishing Old vs. New Physics Scenarios}

Future data which will be obtained with new detector arrays and satellites will give us more clues relating to the origin of the trans-GZK events by distinguishing between the various hypotheses that have been proposed.

An "old physics" zevatron origin will produce air-showers primarily from primaries which are protons or heavy nuclei, with a much smaller number of neutrino-induced showers, the neutrinos being secondaries from CBR photomeson interactions. Zevatron events should cluster near the direction of the sources.

A "new physics" fragger origin mechanism will not produce any nuclei heavier than protons and will produce more ultrahigh energy neutrinos than protons. Thus, it will be important to look for the $\nu$-induced air showers which are expected to originate much more deeply in the atmosphere than proton-induced air showers and are therefore expected to be mostly horizontal showers. Such models also produce more photons than protons. Photons produced in the galactic halo, e.g., from the decay or annihilation of superheavy dark matter, can reach us and will have an anisotropic distribution on the sky. New physics top-down mechanisms may produce harder spectra than are expected from cosmic zevatrons. If differential cosmic ray spectra are parametrized to be of the form $F \propto E^{-\Gamma}$, then for top-down models $\Gamma<2$, whereas for bottom-up models $\Gamma \geq 2$. If Lorentz invariance violation is the explanation for the missing GZK effect, one can also look for the absence of a pair-production $10 \mathrm{EeV}$ "bite" in the spectrum, but this may be more difficult to detect.

1 Other UHECR data are given elsewhere in these proceedings and in Nagano and Watson (2000). 


\section{References}

[1] Ahn, E.-J., Cavaglia, M. and Olinto, A.V. Phys. Letters B (2003) 551, 1.

[2] Alavi-Harati, A. et al., Phys. Rev. Letters 83 (1999) 2128.

[3] Albuquerque, I.F.M. and Baudis, L., Phys. Rev. Letters 90, (2003) 221301.

[4] Allen, S.W.et al., Mon. Not. Roy. Astr. Soc. 346 (2003) 593.

[5] Alfaro, J. and Palma, G., Phys. Rev. D 65, (2002) 103516.

[6] Alfaro, J. and Palma, G., Phys. Rev. D 67, (2003) 083003.

[7] Alfaro, J. et al., Phys. Rev. D 65 (2002) 103509.

[8] Aliosio, R. et al., Phys. Rev. D 62 (2000) 053010.

[9] Amati, L. (2004), e-print astro-ph/0405318

[10] Amelino-Camelia, G. et al., Nature 393 (1998) 763.

[11] Anchordoqui, L.A., Feng, J.L. and Goldberg, H., Phys. Lett. B 535 (2002) 302.

[12] Anchordoqui, L.A. et al., Phys. Rev. D 63 (2001) 124009.

[13] Arkani-Hamed, N. Dimopoulos, S. and Dvali, G.R., Phys. Rev. D 59 (1999) 086004.

[14] Ave, M. et al., Phys. Rev. Letters 85 (2000) 2244.

[15] Barbot, C. and Drees, M., Phys. Letters B 533 (2002) 107.

[16] Berezinsky, V.S., Kachelrieß, M. and Vilenkin, A., Phys. Rev. Letters 79 (1997) 4302.

[17] Berezinsky, V.S., Kachelrieß, M. and Ostapchenko, S., Phys. Rev. D 65 (2002) 083004.

[18] Bhattacharjee, P. and Sigl, G., Phys. Rpts. 327 (2000) 109.

[19] Bhattacharyya, G. et al., Phys. Lett. B564 (2003) (2003) 175.

[20] Biermann, P.L. (1998) in Workshop on Observing Giant Cosmic Ray Air Showers from Space, ed. J.F. Krizmanic, J.F. Ormes and R.E. Streitmatter, (New York: American Institute of Physics, AIP CP-433) p.22.

[21] Biermann, P.L. and Strittmatter, P.A., Astrophys. J. 322 (1987) 643.

[22] Blanton, M., Blasi, P. and Olinto, A.V., Astroparticle Phys. 15 (2001) 275.

[23] Blasi, P., Dick, R. and Kolb, E.W., Astroparticle Physics 18 (2002) 57.

[24] Boldt, E. and Ghosh, P., Mon. Not. Roy. Astron. Soc. 307 (1999) 491.

[25] Boldt, E. and Lowenstein, M., Mon. Not. Roy. Astron. Soc. 316 (2000) L29. 
[26] Bordes, J. et al., Astroparticle Phys. 8 (1998) 135.

[27] Burdman, G., Halzen, F. and Ghandi, R., Phys. Letters B 417 (1998) 107.

[28] Cappellaro, E. et al. (2004) e-print astro-ph/0407261.

[29] Cherepashchuk, A. and Postnov, K., Astron. Rpts. 45 (2001) 517, e-print astro$\mathrm{ph} / 0012512$.

[30] Cheung, D.J.H., Farrar, G.R. and Kolb, E.W., Phys. Rev. D 57 (1998) 4606.

[31] Chisholm, J.R. and Kolb, E.W., Phys. Rev. D 69 (2004) 085001.,

[32] Christensen, L., Hjorth, J. and Gorosabel, J. (2004), e-print astro-ph/0407066,

[33] Cillis, A.N. et al., Phys. Rev. D 59 (1992) 113012.

[34] Coleman, S. and Glashow, S.L., Phys. Rev. D59, (1999) 116008.

[35] De Marco, D., Blasi, P. and Olinto, A.V, Astroparticle Phys., 20 (2003) 53.

[36] Domokos, G. and Kovesi-Domokos, S., Phys. Rev. Letters 82 (1999) 1366.

[37] Domokos, G. and Nussinov, S., Phys. Letters B 187 (1987) 372.

[38] Falcke, H., Rev. Mod. Astron. 14 (2001) 15.

[39] Fargion, D., Mele, B. and Salis, A., Astrophys. J. 517 (1999) 725.

[40] Farrar, G.R., Phys. Rev. Letters 76 (1996), 4111.

[41] Farrar, G.R. and Piran, T., Phys. Rev. Letters 84 (2000) 3527.

[42] Feng, J.L. and Shapere, A.D., Phys. Rev. Letters 88 (2002) 021303.

[43] Ginzburg, V.L. and Ozernoi, L.M., Zh. Eksp. Teor. Fiz. 47 (1964) 1030.

[44] Greisen, K., Phys. Rev. Letters 16 (1966) 748.

[45] Halzen, F. and Hooper, D., Rpts. Prog. Phys. 65 (2002) 1025.

[46] Jacobson, T., Liberati, S., Mattingly, D. and Stecker, F.W., Phys. Rev Letters 93 (2004) 021101.

[47] Jain, P., et al., Physics Letters B 484 (2000) 267.

[48] Kachelrieß, M. and Semikoz, D.V., Phys. Lett. B577 (2003) 1.

[49] Kolb, E.W. and Turner, M.S. (1990), The Early Universe, (Reading: Addison Wesley).

[50] Krolik, J., Astrophys. J. 515 (1999) L73.

[51] Kuz'min, V.A., and Rubakov, V.A., Phys. Atom Nucl. 61 (1998) 1028.

[52] Nagano, M. and Watson, A.A., Rev. Mod. Phys. 72 (2000) 689.

[53] Norris, J., Astrophys. J. 579 (2002) 368. 
[54] Nussinov, S. and Schrock, R., Phys. Rev. D 59 (1999) 105002.

[55] Randall, L. and Sundrum, Phys. Rev. Letters 83 (1999) 3370.

[56] Robinett, R.W., Phys. Rev. D 37 (1998) 84.

[57] Salamon, M.H. and Stecker, F.W., Astrophys. J. 493 (1998) 547 (SS98).

[58] Sarkar, S. and Toldrà, R., Nucl. Phys. B 621 (2002) 495.

[59] Sasaki, N. et al. , Proc. 27th Intl. Cosmic Ray Conf., Hamburg 1 (2001) 333.

[60] Sato, H. and Tati, T., Progr. Theor. Phys. 47 (1972) 1788.

[61] Schmidt, M., Astrophys. J. 552 (2001) 36.

[62] Scully, S.T. and Stecker, F.W., Astroparticle Physics 16 (2002) 271.

[63] Shafi, Q. and Stecker, F.W., Phys. Rev. Letters 53 (1984) 1292.

[64] Shinozaki, K. et al., Astrophys. J. 571 (2002) L117.

[65] Stanev, T. et al., Phys. Rev. Letters 75 (1995) 3056.

[66] Stecker, F.W., Phys. Rev. Letters 21 (1968) 1016.

[67] Stecker, F.W., Astroparticle Phys. 14 (2000) 207.

[68] Stecker, F.W., J. Phys. G, 29 (2003) R48, e-print astro-ph/0309027.

[69] Stecker, F.W. and Glashow, S.L., Astroparticle Phys. 16 (2001) 97.

[70] Stecker, F.W. and Salamon, M.H., Astrophys. J. 512 (1999) 521.

[71] Stecker, F.W. and Scully, S.T. (2004), to be published.

[72] Stern, B.E., Tikhomirova, Ya. and Svensson, R., Astrophys. J. 573 (2002) 75.

[73] Tremaine, S. and Gunn, J.E., Phys. Rev. Letters 42 (1979) 407.

[74] Tyler, C., Olinto, A.V. and Sigl, G., Phys. Rev. D 63 (2001) 055001.

[75] Vietri, M., Astrophys. J. 453 (1995) 883.

[76] Waxman, E., Phys. Rev. Letters 75 (1995) 386.

[77] Weiler, T.J., Phys. Rev. Letters 49 (1982) 234.

[78] Weiler, T.J., Astroparticle Phys. 11 (1999) 303.

[79] Zatsepin, G.T. and Kuz'min, V.A., Zh. Eks. Teor. Fiz., Pis'ma Red. 4 (1966) 144. 